\title{
Extended Stokes parameters for cylindrically polarized beams
}

\author{
Masato Suzuki $\cdot$ Keisaku Yamane $\cdot$ Kazuhiko Oka • \\ Yasunori Toda $\cdot$ Ryuji Morita
}

Received: 16 June 2014/ Accepted: 15 August 2014/Published online: 10 March 2015

(c) The Author(s) 2015. This article is published with open access at Springerlink.com

\begin{abstract}
The extended Stokes parameters for arbitrary cylindrically polarized beams are newly introduced to evaluate their quality. A set of the parameters, expressing a cylindrically polarized state, permits the definition of the degree of polarization that indicates the purity of the spatial symmetry of polarization of a light beam. In addition, the Pancharatnam-Berry phase related to the cylindrically polarized states is described by the new Stokes parameters.
\end{abstract}

Keywords Extended Stokes parameters - Cylindrically polarized beam - Optical vortex . Poincaré sphere .

Pancharatnam-Berry phase

Cylindrically polarized (CP) beams [1] are laser mode beams whose polarization distributions have symmetry of rotation about the beam axis. These beams, including axisymmetrically polarized beams (such as a radially polarized beam) and anti-vortex beams, are promising mode for vector-vortex-mode-division multiplexing in optical communications [2,3]. They have been consequently generated in a lot of studies $[4,5]$ so far. For establishing vectorvortex-mode-division multiplexing technology, quantitative evaluation of the $\mathrm{CP}$ beams is significantly important. Earlier researches have introduced the high-order [6, 7] and the hybrid [8] Stokes parameters to characterize the CP beams. These parameters, however, cannot be responsible

M. Suzuki $(\bowtie) \cdot$ K. Yamane $\cdot$ K. Oka $\cdot$ Y. Toda $\cdot$ R. Morita Department of Applied Physics, Hokkaido University, Kita-13, Nishi-8, Kita-ku, Sapporo 060-8628, Japan

e-mail: masato_suzuki@eng.hokudai.ac.jp

K. Yamane $\cdot$ Y. Toda $\cdot$ R. Morita

JST, CREST, Kita-13, Nishi-8, Kita-ku, Sapporo 060-8628, Japan for the local deviation in the symmetry of CP states, which often appears in experimental measurements. To overcome this issue, we have introduced the extended Stokes parameters (ESPs) for the polarization states with $C_{\infty}$ symmetry in consideration of the local deviation of the symmetry [9]; on the other hand, the CP states other than with $C_{\infty}$ symmetry have not yet been covered.

In the present paper, we extend the coverage of the ESPs so that they describe the CP beams without $C_{\infty}$ symmetry. As a natural extension of the degree of polarization (DOP) from the ordinary Stokes parameters, we define the spatial DOP from the introduced ESPs, which represents a measure in the purity of the symmetry of a $\mathrm{CP}$ beam. Furthermore, we discuss the Pancharatnam-Berry geometric phase [10] for the new ESPs.

We use the following $\mathrm{CP}$ basis to define ESPs:

$\boldsymbol{e}_{r}^{l}=\left(\begin{array}{c}\cos (l \phi) \\ \sin (l \phi)\end{array}\right), \quad \boldsymbol{e}_{\phi}^{l}=\left(\begin{array}{c}-\sin (l \phi) \\ \cos (l \phi)\end{array}\right)$,

where $l$ is an integer value to distinguish the $\mathrm{CP}$ basis, and $\phi$ is the azimuthal angle $\arctan (y / x)$. The $\mathrm{CP}$ basis contains the Cartesian coordinates $(l=0)$ and the cylindrical coordinates $(l=1)$. The $\mathrm{CP}$ basis for $l=1$ and $l \neq 1$ have respectively $C_{\infty}$ and $C_{|l-1|}$ symmetries. The polarization states expressed by the former has been reported in the previous paper [9]; hence, here we describe the polarization states represented by the latter.

A superposition of $l$ th $\mathrm{CP}$ basis can provide circular polarized optical vortex basis:

$\boldsymbol{e}_{ \pm}^{l}=\frac{e^{\mp \mathrm{i} l \phi}}{\sqrt{2}}\left(\begin{array}{c}1 \\ \pm i\end{array}\right)=\frac{\boldsymbol{e}_{r}^{l} \pm \mathrm{i} \boldsymbol{e}_{\phi}^{l}}{\sqrt{2}}$

Here, for a paraxial light wave propagating in $+z$ direction, we decompose its electric field components into the $l$ th 
radial component $E_{r}^{l}$ and the $l$ th azimuthal component $E_{\phi}^{l}$ in $l$ th $\mathrm{CP}$ basis, as expressed by,

$$
\begin{gathered}
\mathbf{E}(r, \phi, z, t)=E_{r}^{l}(r, \phi, z, t) \boldsymbol{e}_{r}^{l}+E_{\phi}^{l}(r, \phi, z, t) \boldsymbol{e}_{\phi}^{l} \\
(r \neq 0 \text { where } l \neq 0) .
\end{gathered}
$$

It is noted that $E_{r}^{l}$ and $E_{\phi}^{l}$ can be described by the sum of $l$ topological-charge Laguerre-Gaussian modes with $p$ th radial index without the phase ramp [1]. A polarization state is not determined at the point of $r=0$ if $l \neq 0$, thus we exclude the $r=0$ point for the decomposition. We define Stokes parameters (SPs) for CP beams, as natural extensions of the ordinary Stokes parameters [11]:

$s_{0}^{l}(r, \phi, z)=\left\langle\left|E_{r}^{l}\right|^{2}+\left|E_{\phi}^{l}\right|^{2}\right\rangle_{\mathrm{T}}$,

$s_{1}^{l}(r, \phi, z)=\left\langle\left|E_{r}^{l}\right|^{2}-\left|E_{\phi}^{l}\right|^{2}\right\rangle_{\mathrm{T}}$,

$s_{2}^{l}(r, \phi, z)=\left\langle E_{r}^{l *} E_{\phi}^{l}+E_{r}^{l} E_{\phi}^{l *}\right\rangle_{\mathrm{T}}$,

$s_{3}^{l}(r, \phi, z)=-\mathrm{i}\left\langle E_{r}^{l *} E_{\phi}^{l}-E_{r}^{l} E_{\phi}^{l *}\right\rangle_{\mathrm{T}}$,

where the symbol $\langle\ldots\rangle_{\mathrm{T}}$ represents the time average. The DOP defined from these parameters $\sqrt{\left(s_{1}^{l}\right)^{2}+\left(s_{2}^{l}\right)^{2}+\left(s_{3}^{l}\right)^{3}} / s_{0}^{l}$ has the same meaning of that of the ordinary $(l=0)$ Stokes parameters; the purity of polarization with respect to time [12]. We refer to this DOP as $\mathcal{P}_{l}^{\text {SPs }}$ defined from $l$ th SPs.

Now we introduce our new ESPs, defined in the following:

$S_{i, l}^{\mathrm{E}}(z)=\iint_{A} s_{i}^{l}(r, \phi, z) r \mathrm{~d} r \mathrm{~d} \phi \quad(i=0-3)$,

which are the integrals of SPs in the beam cross-section. Their physical significance will be discussed later. The area $A$ should be large enough to cover the entire beam. Table 1 summarizes the notation of various Stokes parameters.

These parameters are useful for analyzing polarization distribution of a laser beam in its cross-section. We have discussed linear and nonlinear propagation of an axisymmetrically polarized mode with $C_{\infty}$ symmetry in a uniaxial crystal along its optic axis in ref. [9].

Our ESPs define the spatially averaged DOP that takes into account of the purity of the cylindrical symmetry,

Table 1 Notation of various Stokes parameters $(i=0-3)$

$s_{i}^{0}(r, \phi, z) \quad$ The ordinary Stokes parameters at $(r, \phi, z)$

$s_{i}^{l}(r, \phi, z) \quad$ The Stokes parameters (SPs) for CP beams at $(r, \phi, z)$

$S_{i, l}^{\mathrm{E}}(z) \quad$ The extended Stokes parameters (ESPs) whereas the high-order [6, 7] and the hybrid [8] Stokes parameters are for the perfectly symmetrical polarization states. In this part, we describe the coherency matrix consisting of time and space averages of electric field components. This coherency matrix gives the new DOP from the ESPs in the same way that the ordinary coherency matrix provides the definition of DOP $\mathcal{P}[12,13]$.

The coherency matrix for $l$ th $\mathrm{CP}$ basis (hereafter, the $l$ th coherency matrix) is written by the following equation,

$J_{l}(z)=\left(\begin{array}{cc}J_{r r}^{l}(z) & J_{r \phi}^{l}(z) \\ J_{\phi r}^{l}(z) & J_{\phi \phi}^{l}(z)\end{array}\right)$.

In the manner similar to the conventional coherency matrix, the components of the $l$ th coherency matrix are defined by $J_{i j}^{l}(z)=\left\langle E_{i}^{l} E_{j}^{l *}\right\rangle_{\mathrm{TS}}(i, j=r, \phi)$, where the bracket $\langle\ldots\rangle_{\mathrm{TS}}$ means time and space $((r, \phi)$ plane $)$ average:

$$
\langle f(r, \phi, z, t)\rangle_{\mathrm{TS}}=\iint_{A}\langle f(r, \phi, z, t)\rangle_{\mathrm{T}} r \mathrm{~d} r \mathrm{~d} \phi / \iint_{A} r \mathrm{~d} r \mathrm{~d} \phi .
$$

In the same way that the ordinary DOP is written as $\mathcal{P}=$ $\sqrt{1-4 \operatorname{det}[J] /(\operatorname{tr}[J])^{2}}[12,13]$, the DOP from $l$ th ESP $(l$ th DOP) is consequently defined by

$$
\mathcal{P}_{l}=\sqrt{1-4 \frac{\operatorname{det}\left[J_{l}\right]}{\left(\operatorname{tr}\left[J_{l}\right]\right)^{2}}} .
$$

Here, substituting the relationships $\operatorname{tr}\left[J_{l}\right]=S_{0, l}^{\mathrm{E}}$ and $4 \operatorname{det}\left[J_{l}\right]=\left(S_{0, l}^{\mathrm{E}}\right)^{2}-\left(S_{1, l}^{\mathrm{E}}\right)^{2}-\left(S_{2, l}^{\mathrm{E}}\right)^{2}-\left(S_{3, l}^{\mathrm{E}}\right)^{2}$, we obtain

$$
\mathcal{P}_{l}=\sqrt{\left(S_{1, l}^{\mathrm{E}}\right)^{2}+\left(S_{2, l}^{\mathrm{E}}\right)^{2}+\left(S_{3, l}^{\mathrm{E}}\right)^{2}} / S_{0, l}^{\mathrm{E}} .
$$

This is the analogous formula to that for ordinary DOP. In the same way that the ordinary DOP originating from time average relates to the purity of polarization with respect to time [12], the $l$ th DOP relates to that with respect to time and space. If the electric field exhibits temporally complete polarization in the $(r, \phi)$ plane, the $l$ th DOP gives the purity of polarization with respect to space (Table 2 ). The $l$ th DOP, however, contains a temporally unpolarized state when the beam is not a state with temporally complete polarization.

Thus, we introduce the $l$ th DOP for the spatial distribution (DOP-SD) $\mathcal{P}_{l}^{\text {space }}$ as follows: [14]

$$
\mathcal{P}_{l}^{\text {space }}=\sqrt{\left(S_{1, l}^{\mathrm{E}}\right)^{2}+\left(S_{2, l}^{\mathrm{E}}\right)^{2}+\left(S_{3, l}^{\mathrm{E}}\right)^{2}} / S_{0, l}^{\mathrm{E},(\mathrm{P})},
$$

where $S_{0, l}^{\mathrm{E},(\mathrm{P})}$ is the integral of the amount proportional to the time-averaged intensity of light with temporally complete polarization [15]: 
Table 2 Comparison of DOP

\section{DOP $\mathcal{P}$}

DOP from ESPs $\mathcal{P}_{l}$

DOP-SD from ESPs $\mathcal{P}_{l}^{\text {space }}$

DOP from higher order $[6,7]$ or hybrid [8] Stokes parameters

$S_{0, l}^{\mathrm{E},(\mathrm{P})}=\iint_{A} \sqrt{\left(s_{1}^{l}\right)^{2}+\left(s_{2}^{l}\right)^{2}+\left(s_{3}^{l}\right)^{2}} r \mathrm{~d} r \mathrm{~d} \phi$.

$\mathcal{P}_{l}^{\text {space }}$ is a measure of spatial polarization uniformity in the lth CP basis.

The DOP defined from the ESPs is an important value for both generating and using $\mathrm{CP}$ modes, providing the purity or the quality factor of the polarization distribution.

Poincaré sphere, which is the spherical surface occupied by completely polarized states in the space of the conventional normalized Stokes vector, is useful for describing polarization states visually. In analogy to the conventional DOP, the new DOP $\mathcal{P}_{l}$ from ESPs represents the length of the normalized extended Stokes vector $\left(\tilde{S}_{1, l}^{\mathrm{E}}, \tilde{S}_{2, l}^{\mathrm{E}}, \tilde{S}_{3, l}^{\mathrm{E}}\right)$, of which components are $\tilde{S}_{i, l}^{\mathrm{E}}=S_{i, l}^{\mathrm{E}} / S_{0, l}^{\mathrm{E}}(i=1-3)$. Figure 1 shows typical CP states on the Poincare sphere for $l=1$ and $l=-1$ ESPs. Thus, the states with spatially and temporally complete polarization are only on the surface of Poincaré sphere for ESPs and partially polarized states with respect to time or (and) space are inside the surface.

The Pancharatnam-Berry phase $[10,16,17]$ is an additional phase factor when a quantum (such as a photon and an electron) traveled along a closed loop in a state space, which is first discussed by Pancharatnam [10]. Reference 18 has experimentally shown that the Pancharatnam-Berry phase shift occurs in optical systems using waveplates and polarizers. A light beam undergoes a phase shift which is half of the solid angle $\Omega$ subtended to a closed path on the Poincaré sphere [10, 18].

Here, we discuss the Pancharatnam-Berry phase for the $\mathrm{CP}$ states. We consider an optical system possessing waveplates and polarizers which are $q\left(=l_{\mathrm{WP}}\right)$-plates [19]: $J_{n}^{l_{\mathrm{WP}}}=\left\{\begin{array}{c}U_{\theta_{n}}^{l_{\mathrm{WP}}}\left(e^{\mathrm{i} \psi_{n}^{\mathrm{F}}} \boldsymbol{e}_{r}^{l_{\mathrm{WP}}}+e^{\mathrm{i} \psi_{n}^{\mathrm{s}}} \boldsymbol{e}_{\phi}^{l_{\mathrm{WP}}}\right) U_{-\theta_{n}}^{l_{\mathrm{WP}}}(\text { waveplate }), \\ U_{\theta_{n}}^{l_{\mathrm{WP}}} \boldsymbol{e}_{r}^{l_{\mathrm{WP}}} U_{-\theta_{n}}^{l_{\mathrm{WP}}}(\text { polarizer }),\end{array}\right.$

where $n(\geq 1)$ is a waveplate or polarizer number, $J_{n}^{l_{\mathrm{WP}}}$ is a Jones matrix of the $n$th waveplate or polarizer, $\psi_{n}^{\mathrm{F}}$ and $\psi_{n}^{\mathrm{S}}$ are the phase retardances on the fast and slow axes of the $n$th waveplate, and $\theta_{n}$ is a rotation angle of the $n$th waveplate or polarizer. $U_{\theta}^{l}$ is a rotation matrix defined by

$U_{\theta}^{l}=\left(\begin{array}{cc}\cos \theta & -\sin \theta \\ \sin \theta & \cos \theta\end{array}\right)$.

After a light beam propagates through the $n_{\max }$ waveplates and/or polarizers, the electric field vector $\tilde{\mathbf{E}}\left(r, \phi, z=z_{\text {out }}\right)$ is

$\tilde{\mathbf{E}}\left(r, \phi, z=z_{\text {out }}\right)=\prod_{n=1}^{n_{\max }} J_{n_{\max }-n+1}^{l_{\mathrm{WP}}} \tilde{\mathbf{E}}\left(r, \phi, z=z_{\text {in }}\right)$,

where $\tilde{\mathbf{E}}\left(z=z_{\text {in }}\right)$ is the initial electric field vector and $\prod_{n=1}^{n_{\max }} J_{n_{\max }-n+1}^{l_{\mathrm{WP}}}=J_{n_{\max }}^{l_{\mathrm{WP}}} J_{n_{\max }-1}^{l_{\mathrm{WP}}} \cdots J_{1}^{l_{\mathrm{WP}}}$.

On the assumption that the electric field is monochromatic as $\mathbf{E}(r, \phi, z, t)=\tilde{\mathbf{E}}(r, \phi, z) \exp (\mathrm{i}(k z-\omega t))$, where $\omega$
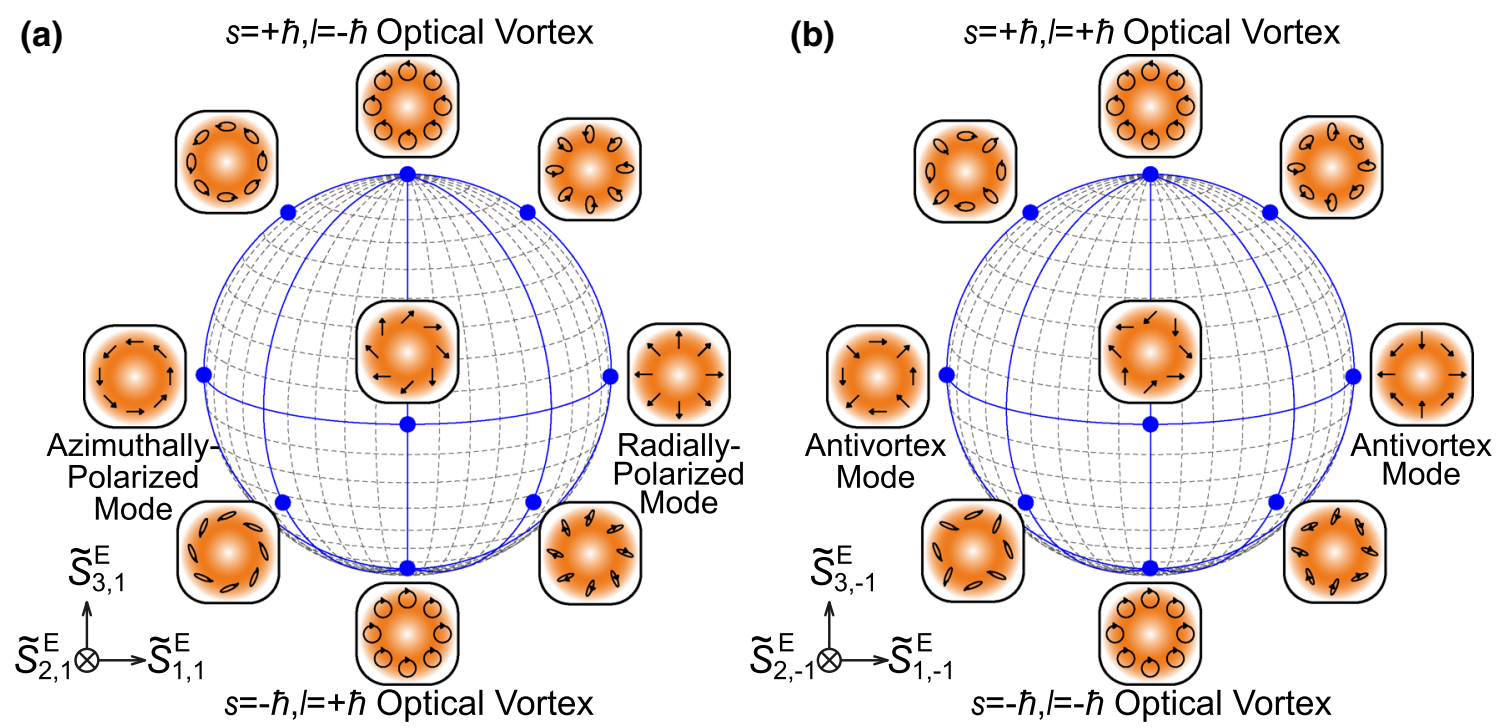

Fig. 1 Poincaré sphere corresponding to the $\mathbf{a} l=1$ and $\mathbf{b} l=-1$ ESPs, and typical polarization states on blue points (color online). 
Fig. 2 A typical example of the Pancharatnam-Berry phase for $\mathrm{CP}$ beams. a An experimental configuration. b A corresponding trajectory on the Poincaré sphere for $l=-1$ ESPs (color online)

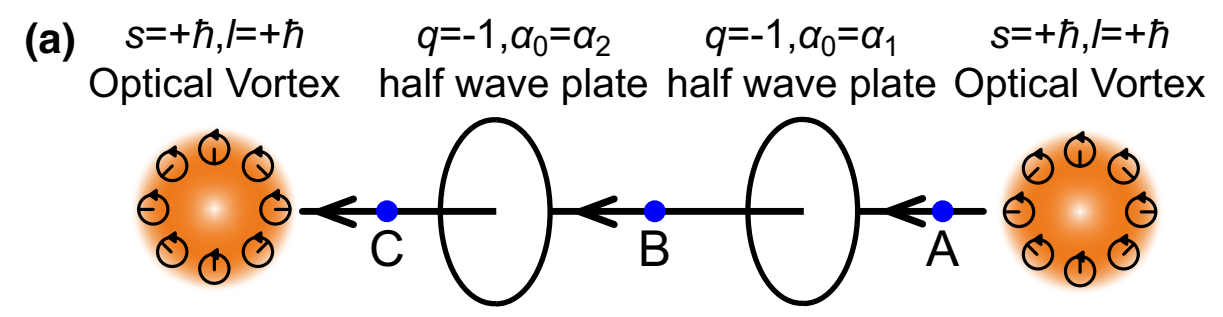

(b)

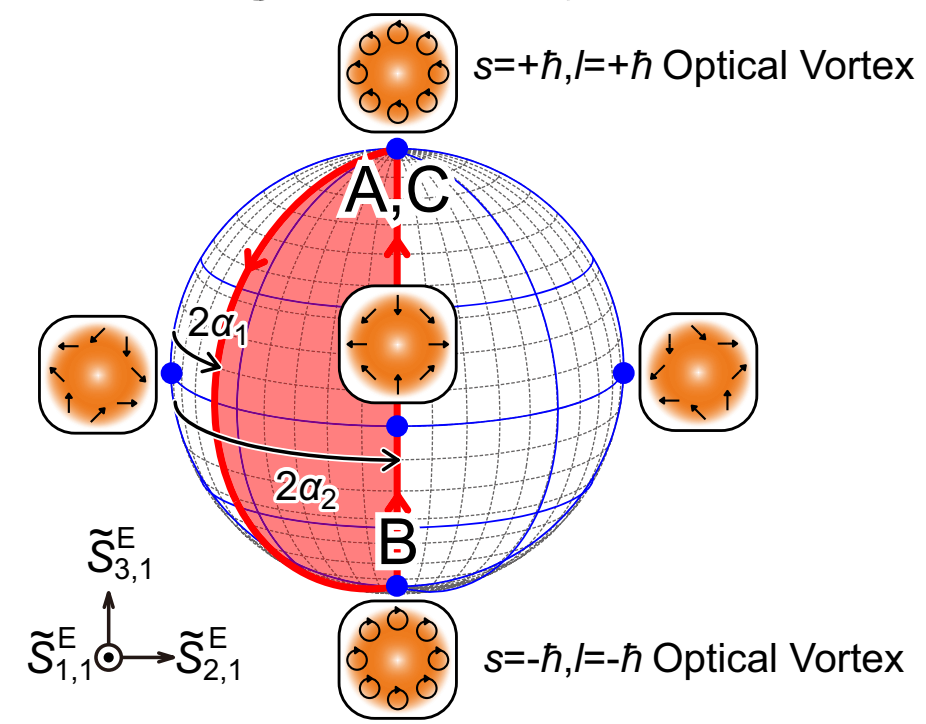

and $k$ are respectively the angular frequency and the propagation constant at $\omega$, the DOP $\mathcal{P}_{l_{\mathrm{WP}}}$ is conserved through the propagation of $z_{\text {in }} \leq z \leq z_{\text {out }}$ for the light of which $\mathcal{P}_{l_{\mathrm{WP}}}$ is 1 at $z=z_{\text {in }}$.

This means that the symmetry of the polarization distribution is conserved and the point $\left(\tilde{S}_{1, l}^{\mathrm{E}}, \tilde{S}_{2, l}^{\mathrm{E}}, \tilde{S}_{3, l}^{\mathrm{E}}\right)$ draws a trajectory on the surface.

Since the normalized extended Stokes vectors are subjected to the same mathematics as the normalized conventional Stokes vector, the Pancharatnam-Berry phase $\phi_{\mathrm{PBP}}^{l_{\mathrm{WP}}}$ shift is given by

$\phi_{\mathrm{PBP}}^{l_{\mathrm{WP}}}=-\frac{\Omega}{2}$,

when $\tilde{S}_{i, l_{\mathrm{WP}}}^{\mathrm{E}}\left(z_{\text {in }}\right)=\tilde{S}_{i, l_{\mathrm{WP}}}^{\mathrm{E}}\left(z_{\text {out }}\right)$.

Figure 2 shows a typical example of the PancharatnamBerry phase for CP beams. An $s=+\hbar, l=+\hbar$ optical vortex beam [20] passes through two $q(=-1)$ half-wave plates; the one for $q=-1, \alpha_{0}=\alpha_{1}$, and the other for $q=-1, \alpha_{0}=\alpha_{2}$ (Fig. 2(a); $q$ and $\alpha_{0}$ are defined in ref. 19). Conserving the $C_{2}$ symmetry of the polarization distribution, the beam experiences the Pancharatnam-Berry phase $\phi_{\mathrm{PBP}}^{l_{\mathrm{WP}}}=$ $2\left(\alpha_{1}-\alpha_{2}\right)$, which corresponds to the half of the solid angle subtended to the trajectory of the beam on the Poincare sphere for $l=-1$ ESPs (Fig. 2(b)).

We introduced the ESPs for arbitrary CP beams with $C_{|l-1|}$ symmetry in addition to the previous report for those with $C_{\infty}$ symmetry. Using the introduced parameters, we defined the spatial DOP and discussed the Pancharatnam-Berry phase in the analogy to the conventional Stokes parameters.

The ESPs are of use for evaluating the properties of $\mathrm{CP}$ laser beams. While spatial polarization properties were so far qualitatively estimated by monitoring the intensity distribution after passing through a polarizer, measuring our ESPs and thereby obtaining the spatial DOP, which are related to the purity of the spatial symmetry of the $\mathrm{CP}$ beams, give the qualitative information of the polarization distribution. Our ESPs and DOP are crucial for applications such as laser processing [21] and spectroscopy [22] using CP modes.

Open Access This article is distributed under the terms of the Creative Commons Attribution License which permits any use, distribution, and reproduction in any medium, provided the original author(s) and the source are credited.

\section{References}

1. A.A. Tovar, J. Opt. Soc. Am. A 15, 2705 (1998)

2. S. Ramachandran, P. Kristensen, M.F. Yan, Opt. Lett. 34, 2525 (2009)

3. N. Bozinovic, Y. Yue, Y. Ren, M. Tur, P. Kristensen, H. Huang, A.E. Willner, S. Ramachandran, Science 340, 1545 (2013)

4. Q. Zhan, Adv. Opt. Photon. 1, 1 (2009) 
5. C. Maurer, A. Jesacher, S. Fürhapter, S. Bernet, M. Ritsch-Marte, New J. Phys. 9, 78 (2007)

6. G. Milione, H.I. Sztul, D.A. Nolan, R.R. Alfano, Phys. Rev. Lett. 107, 053601 (2011)

7. G. Milione, S. Evans, D.A. Nolan, R.R. Alfano, Phys. Rev. Lett. 108, 190401 (2012)

8. A. Holleczek, A. Aiello, C. Gabriel, C. Marquardt, G. Leuchs, Opt. Express 19, 9714 (2011)

9. M. Suzuki, K. Yamane, K. Oka, Y. Toda, R. Morita, Opt. Express 22, 16903 (2014)

10. S. Pancharatnam, Proc. Indian Acad. Sci. Sect. A 44, 247 (1956)

11. E. Hecht, Optics, 4th edn, Chap. 8 (Addison-Wesley, 2002)

12. J.W. Goodman, Statistical optics, Chap. 4 (Wiley, New York, 1985)

13. E. Wolf, Nuovo Cimento 13, 1165 (1959)

14. Subtraction of the temporally unpolarized state from the denominator of Eq. (12) is needed
15. D.H. Goldstein, Polarized light, 3rd edn, Chap. 5 (CRC Press, USA, 2011)

16. M.V. Berry, Proc. R. Soc. Lond. A 392, 45 (1984)

17. Y. Aharonov, J. Anandan, Phys. Rev. Lett. 58, 1593 (1987)

18. R. Bhandari, J. Samuel, Phys. Rev. Lett. 60, 1211 (1988)

19. L. Marrucci, C. Manzo, D. Paparo, Phys. Rev. Lett. 96, 163905 (2006)

20. L. Allen, M.W. Beijersbergen, R.J.C. Spreeuw, J.P. Woerdman, Phys. Rev. A 45, 8185 (1992)

21. C. Hnatovsky, V. Shvedov, W. Krolikowski, A. Rode, Phys. Rev. Lett. 106, 123901 (2011)

22. Y. Tokizane, K. Shimatake, Y. Toda, K. Oka, M. Tsubota, S. Tanda, R. Morita, Opt. Express 18, 2144 (2010) 